\title{
EVALUATION OF KNOWLEDGE, ATTITUDES AND BEHAVIOURS ABOUT COVID-19 AMONG CANCER PATIENTS
}

\author{
Seher Nazlı Kazaz ${ }^{1}$ and Atila Yıldırım ${ }^{1}$ \\ ${ }^{1}$ Karadeniz Technical University Faculty of Medicine
}

December 30, 2020

\begin{abstract}
Background COVID-19 pandemic is experienced intensely in our country like others. The presence of cancer in the patients is associated with morbidity and mortality in the course of COVID-19 disease. In this study, we aimed to evaluate the level of knowledge, attitude and behaivours regarding coronavirus disease in cancer patients. Methods This study is a single-center study which consists of 220 cancer patients who treated in our clinic. A questionnaire which consisted of three parts measuring the knowledge, attitudes and behaviours among cancer patients about COVID-19 was completed by each patient face-to-face during June, 2020. Results Most patients were familiar with the general information on COVID-19. Most cancer patients were aware of the symptoms of COVID-19 and that they were at greater risk. In $50 \%$ of the patients participating in the survey, it was observed that the covid-19 outbreak did not thought delaying cancer treatments. Half of the cancer patients who didn't receive active cancer treatment preferred to have control tests performed at the time deemed appropriate by their doctors during the pandemic. $38.6 \%$ of them wanted to have control examinations done on the scheduled time. All of the patients wore masks when going out during the outbreak. Nearly all of the participants (99\%) washed their hands to prevent contamination. $95 \%$ of the participants paid attention to social distance with other people. Contributors in the questionnaire with higher educational level had significantly higher knowledge mean scores compared to those with others. Conclusion In general, cancer patients are conscious about pandemic. They move with high compliance with their doctors in this period. Since COVID-19 infection is more severe in cancer patients, it is very important to evaluate these parameters to increase their knowledge and to correct their incomplete or incorrect information about this disease.
\end{abstract}

\section{EVALUATION OF KNOWLEDGE, ATTITUDES AND BEHAVIOURS ABOUT COVID-19 AMONG CANCER PATIENTS}

Seher Nazlı Kazaz, Atila YILDIRIM

Department of Medical Oncology, Faculty of Medicine, Karadeniz Technical University, Trabzon, Turkey

\section{INTRODUCTION}

The severe acute respiratory syndrome coronavirus 2 (SARS-CoV-2), which caused the pandemic of COVID19 (Coronavirus disease-2019), started in December 2019 in Wuhan city, China, affecting the whole world, causing the death of thousands of people (1). This pandemic was recognized by the World Health Organization on January 30, 2020 (2). The first identified COVID-19 case of the worldwide COVID-19 outbreak in Turkey was announced by the Ministry of Health on March 11, 2020. As of 18th December 2020, the World Health Organization reported 73275943 confirmed cases, with 1650348 deaths from SARS-CoV-2 related disease (coronavirus disease 2019 [COVID-19]). Of them, 1955680 cases and 17364 deaths have been described in Turkey. 
The virus is transmitted to people through airborne droplets by contact with an infected person or contaminated surface (3). The main clinical symptoms of the disease are fever, dry cough, fatigue, muscle pain, and dyspnea. In severe cases, this virus causes deadly pneumonias leading to acute respiratory failure syndrome (4). Elderly people and patients with comorbidity are more likely to become infected. In addition, serious complications such as acute respiratory distress syndrome (ARDS) are observed more frequently in these individuals (5). Cancer is a chronic disease and cancer patients have lower immune systems than other normal people because of both cancer and the immunosuppressive drugs they take, which makes them more susceptible to infections (6). Accordingly, we can say that cancer patients infected with SARS-CoV-2 corona virus are more risky than other populations. In this context, cancer care was immediately established as a heath priority by the National Medical Council and the Ministry of Health.

In one study, the disease was more severe in cancer patients than in non-cancer patients (7). In another study by Mengyuan Dai et al., compared to COVID-19 patients with and without cancer, the mortality risk is 2.3 times, the need for intensive care units is 2.8 times and the risk of developing at least one serious or critical symptom is 2.8 times were found higher in cancer patients. However, utilization of invasive mechanical ventilation was determined higher in patients with cancer (8).

There has been no proven treatment or vaccine against Coronavirus pandemic. However, it is known that strong infection control measures should be taken as the primary intervention to prevent the spread of the disease (9). As in other countries fighting pandemics, strict measures are being taken in Turkey. The first of these is to inform the community with television, newspapers, social media or health care workers. Measures taken in Turkey include suspension of public transport, closure of public spaces such as shopping malls, close management of communities, isolation of infected or suspicious cases. It is very important that cancer patients, along with people in the community, comply with the control measures against COVID-19 and this situation is affected by the knowledge, attitudes and behaviors of COVID-19.

The ESMO guidelines regarding the management of cancer patients during the COVID-19 outbreak, priority should be given to adjuvant therapies in patients with resected high-risk disease, which is expected to achieve a significant absolute survival benefit. Similarly, the benefits and risks of palliative treatments during pandemic should be discussed. "Treatment holidays", "Stop and Go", maintenance and transition to oral medications, if available, should be considered.

Cancer patients who often have to come to the hospital in this process are at increased risk of transmission. At the same time, their mortality and morbidity increase due to the impact of their immunity in relation to the treatments they receive. Due to these risks, it is very important that cancer patients survive this period with minimal damage. In order to comply with the pandemic measures of patients and also to increase compliance with cancer treatment, it is necessary to properly implement the measures and to be properly informed by the primary physicians of the patients about the risks associated with cancer treatment. In this study, we aimed to evaluate the level of knowledge, attitudes and practices about COVID-19 cancer patients who were followed in our clinic during this period when the COVID-19 outbreak was rising rapidly in our country. At the same time, this study will allow our patients to be reininformed and corrected about incomplete or misobtained information.

\section{SUBJECTS AND METHODS}

\section{Study Population}

Between June 1 and July 1, 2020, patients with various types of cancer who applied to KTU School of Medicine, Medical Oncology Outpatient Clinic were included in the study. Our study is an observational, single-center study consisting of 220 patients. An average of 25 patients who came to our daily outpatient clinic were calculated and 400 patients admitted in a total of one month were predicted. $50 \%$ frequency was taken into account for situations whose frequency is unknown; approximately 220 patients were interviewed in the $95 \%$ confidence interval.

\section{Study Tool}


Apart from the demographic characteristics of patients, our own survey study consisting of 25 questions was planned. The survey was designed by the doctor as a Turkish native language. The survey consisting of 25 questions in total was outlined in 3 sections. In the first section, patients were asked general information about COVID-19. In the second section, questions were raised about their thoughts on prevention methods. In the third part, questions were asked about the COVID-19 outbreak and cancer. Except for 2 questions in the survey, the answers were designed to consist of 3 options. In order to be easy and clear, the majority of options were created as "yes, no or not sure". Knowledge on the subject of COVID-19 was evaluated by answering 11 multiple-choice questions followed by the calculation of a total cumulative knowledge score for every contributor. Questions were given one point for correct response and zero point for incorrect answers or unsure answers. The maximum score was 11 , and the minimum was 0 . Interviews were conducted in the outpatient room taking into account the rules of pandemic isolation and social distance with patients applying for routine control or treatment process. The purpose of the study and absolute confidentiality of personal information was explained to the patients by the attending physician. Patients who were over 18 years of age, who had normal intelligence levels as clinical observation and who did not have organic brain syndrome, who were not in the terminal period of cancer, and who agreed to the study were included in the study. Individuals with brain metastasis or primary brain tumors were not included in the study.

\section{Validation and Pilot Study}

A prelimanary phase was made to evaluate the validity and reliability of the questionnaire prior to its use. Primarily, two Turkısh specialists in the subject area of epidemiology and research in Turkısh universities were asked to evaluate the grade to which questions in the questionnaires are relevant regarding COVID-19. Also the survey questions were modified and edited accordingly.

The survey was pre-tested on 15 participants excluded from the study sample. The patients were requested to fill the questionnaire twice one week apart. Information were used to evaluate internal consistency reliability using cronbach's alpha. The cronbach's alpha value of the pilot study was 0.90 and the intra-class correlation coefficient was 0.83 . That is, results showed sufficient internal consistency reliability.

\section{Sampling}

The sample size was calculated by using the openEpi (Open source epidemiologic Statistics for Public Health) soft-ware. At $95 \%$ confidence interval, the calculated sample size was 197 contributors. However, we included 200 participants in the study. And the interviews were stopped at the end of the first month.Formun Üstü

\section{Statistical Analysis}

"Statistical Package for Social Science (SPSS) 23.0" program is used for the analyses. Descrictive statistical analysis was aplied for identify the items included in the questionnaire. While applying the analyses, we presented continuous numeric variables with normal distribution as mean \pm standard deviation (SD). Also, those without normal distribution as median, minimum, and maximum values and percentages were used to describe the categorical data. The suitability of the data for normal distribution was analyzed by the Kolmogorov-Smirnov test. Comparison of numerical variables between independent groups was analyzed using the Mann Whitney $\mathrm{U}$ test and the Kruskal Wallis test because the normal distribution condition was not met. The Bonferroni test from the posthoc test statistics was used to determine the source of the significant difference between the groups. We admitted values of $\mathrm{p}<0.05$ in the comparison results between the groups as statistically significant.

\section{Ethical Considerations}

An application of approval for our study was made to the medical faculty ethics committee of Karadeniz Technical University on 05.05.2020, however due to the pandemic conditions the committee was able to give the approval on 03.07.2020. Afterwards, the surveys were completed quickly and our study was conducted. The submission of the answered questionnaire was regarded consensual to participate in the study.

\section{RESULTS}




\section{Participants' characteristics}

A total 220 patients completed the survey questionnaire. The demographic characteristics of the study participants are shown in Table 1. Of all the patients, 108 (49.1\%) were female and $112(50.9 \%)$ were male. $4.5 \%$ of the patients in the study were between 18 and 35 years, $19.5 \%$ were between 36 and 50 years, around half the patients $(54,1 \%)$ aged between 51 to 65 and $21.8 \%$ aged above 65 years. Most of the patients were married and this rate was $90.5 \%$. The majority of the patients involved in the study lived in the family home $(98.2 \%)$, while the rest lived alone (1.8\%). $6.4 \%$ of the patients who participated in the questionnaire had no literacy; about half $(49.5 \%)$ were primary school graduates, $30 \%$ were middle-high school graduates and $14 \%$ were university graduates. While $85 \%$ of the patients who participated in the study were not working, $15 \%$ were active working. Table 1 also establishes that \%48.6 of the individuals had a history of one or more chronic illnesses.

Most of the diagnoses the patients had were gastrointestinal cancers $(33.2 \%)$ and breast cancer $(26.8 \%)$. Additionally, $41 \%$ of these patients were metastatic; $59 \%$ were nonmetastatic. The ECOG performance scale of $87 \%$ of patients was $0-1$; the rest $2-3$.

\section{General information about COVID-19 and considerations on COVID-19 prevention measures}

$72.7 \%$ of participants claimed that they had never heard the pandemic word before, while $24 \%$ said they had heard it before. The most commonly expressed sources of knowledge were the written and visual media (TV, newspaper etc.) (89\%), the internet (website, social media etc.) $(6.8 \%)$ followed by the doctors $(4.2 \%)$ (Table 2).

The first part of our survey is information questions about the COVID-19 outbreak, as the first two questions in this section are questions based on interpretation; these first two questions are not included in the knowledge score. The information score includes 5 questions in the first section and 6 questions about COVID-19 protection in the second section. As a result, we directed patients to eleven knowledge questions about the COVID-19 pandemic. These questions are shown in table 3. When calculating the knowledge score, only correct answers were accepted from the three options. The total knowledge score varied between 3 to 11, with a mean of $9.88 \pm 1.55$. Almost all the participants answered the question about where the outbreak first appeared correctly. The majority of the patients $(87.3 \%)$ reported that the virus had spread through droplets. $90 \%$ of patients reported fever, cough and fatigue as symptoms. $90 \%$ of patients said yes to the question of whether most patients could survive this infection with early treatment. The majority (85.9\%) said yes to the question about whether COVID-19 disease can be more serious in those with chronic disease such as advanced age, diabetes mellitus, hypertension and cancer; $11.4 \%$ said they were unsure. $99 \%$ of the patients answered the question correctly that they should avoid crowded places and close contact with other people. While $85 \%$ of the patients who participated in the survey thought it is beneficial to wear a mask to prevent the transmission of the virus, $10 \%$ reported that it was not useful. $68.6 \%$ of the patients correctly answered the question of whether people with COVID-19 can transmit the virus to others when they do not have a fever and cough. While $16.8 \%$ stated that they were not sure; $14.5 \%$ said it cannot be transmitted. Our question is that children and young adults are not affected by COVID-19, so they do not need to take action; while $86.4 \%$ stated that this statement was wrong. The majority of patients $(99.1 \%)$ responded correctly to our question that hands should be washed at least 20 seconds to prevent contamination. $95.5 \%$ of the patients answered the question as to how many days the people who come into contact with someone who is infected with the coronavirus should be observed in quarantine, saying fourteen days.

\section{COVID-19 Outbreak and Cancer Related Questions}

$87.7 \%$ of the participants thought that COVID-19 disease would progress more seriously in cancer patients, $10 \%$ said they were not sure. During the COVID-19 epidemic period, half of the cancer patients who were in control left the time of the control tests to their doctors, while $38.6 \%$ stated that their examinations should be done on time, although there is a risk of transmission. Half of the cancer patients who participated in the questionnaire thought that the COVID-19 outbreak did not prevent the treatment of cancer patients from continuing. However, $27.3 \%$ thought it was an obstacle. While half of patients were concerned about 
the continuation of cancer treatment during the outbreak, half expressed no concern. The majority of those who were concerned expressed that they were more afraid of the COVID-19 transmission than disruption of their cancer treatments. The majority of patients ( $70 \%)$ said they did not think about our question about whether you thought of delaying your cancer treatment. When asked whether your oncology doctor informed you about COVID-19 disease, $48.7 \%$ of patients said they informed us, $42 \%$ said they did not inform us. All of the patients said they wore masks when they left the house during the outbreak. The majority of patients $(72.7 \%)$ stated that they came to the hospital with a companion, while $14.5 \%$ said they came alone. About $95 \%$ of patients stated they were paying attention to social distance with other people. While $82.7 \%$ of the patients thought that they took the necessary precautions to protect against the disease, $15 \%$ stated that they applied partially. During the period of the COVID-19 outbreak, the majority of cancer patients (60.9\%) expressed that they did not receive supportive products (Table 4.1 and 4.2).

The relation between socio-demographic characteristics and knowledge about COVID-19 is demonstrated in table 5. Nearly similar knowledge mean scores were observed for male and female participants $(9.93 \pm$ 1.54 and $9.88 \pm 1.56)$ with no statistically signifant difference. Also there were no statistically significant differences in information scores according to age groups and marital status ( $\mathrm{p}>0.05)$. In the same way, there was no statistical difference in information scores according to their marital status $(\mathrm{p}=0.06)$. The knowledge mean scores were significantly related to the level of education $(\mathrm{p}<0.001)$. Contributors in the survey with university or higher education had significantly higher knowledge mean scores compared to those with lower levels of education. There was no statistically significant difference between the participants' knowledge score and who they lived with and whether they were actively working or not $(\mathrm{p}>0.05)$.

\section{DISCUSSION}

COVID-19 disease was discovered during the outbreak of severe pneumonia in Wuhan, China, in December 2019 (10). Afterwards, this COVID-19 outbreak spread to become a global pandemic. Many cancer patients often visit the hospital for disease monitoring and treatment. Cancer patients can be immunocompromised due to the underlying malignancy or anticancer therapy, so they are at higher risk of developing infections, like COVID-19 disease.

This survey provides an insight on the level of knowledge, attitudes and behaviours of our cancer patients on infection control with a special emphasis on COVID-19. However, this study will allow our patients to be reininformed and corrected about incomplete or misobtained information.

In our study, the numbers of men and women surveyed were almost equal (respectively, 112 vs 108). In addition, more than half of the age of patients was over the age of 50 . This may be due to the fact that adult cancers increase with age.

\section{Knowledge and attitudes of participants about COVID-19}

In general terms, patients in our questionnaire had good main knowledge about the COVID-19 disease. There was no statistically significant difference between the mean knowledge score and age, sex, marital status, with whom the patient lived, their occupation, stages of cancer. However, in our contributors, the knowledge score was importantly higher among higher educational levels. Patients surveyed with university or higher education had significantly higher knowledge mean scores compared to those with lower levels of education. In the study conducted by Zhong et al., the knowledge score of the participants about COVID-19 was measured in an online cross-sectional survey, in which 6910 people participated and statistically significant difference was found between education level and knowledge level (11). Also in the study conducted by Erdem D. and Karaman I., the knowledge of the coronavirus transmission route they asked was found to be correlated with the increase in education level (12). $72.7 \%$ of participants claimed that they had never heard the pandemic word before, this may be related to the educational level of the participants. Because, more than half of our patients had a low education level. Written and visual media including TV and newspapers represented the most important sources of information. The most frequently referenced source of information about COVID19 in the study conducted with a healthy population in Egypt; social media platforms and the internet (13). In an another study in our country, which searching the information sources of cancer patients' relatives, 
television was the rare referred information source about cancer, unlikely. In the same study, the internet was the second most referenced information source about cancer by family caregivers following the oncologists. (14). The reason for this may be that our patients participating in survey, had a low educational level, so they can not use the internet and social media effectively.

At the question we asked about where the COVID-19 started, almost all participants answered the question correctly about where the outbreak first appeared and spreaded the world and our country. When we asked about the the transmission way, most of the patients (87.3\%) answered correctly, saying with respiratory droplets. Erdem D. and Karaman I. in their study, this rate was $48.3 \%$ (12). Also the majority of participants reported that fever, dry cough, fatigue and muscle pain are the main symptoms. These results were similar to the study among Jordanian dentists about awareness, perception, and attitude regarding COVID-19 and infection control (15). A vast majority of contributors believed that the disease is more serious for the patients who have diabetes mellitus, hypertension and cancer diseases and elderly patients. This has been proven from the study published about the COVID-19 disease in China. In this study by Mengyuan Dai et al., compared to COVID-19 patients with and without cancer, the mortality risk is 2.3 times higher for cancer patients (8).

Almost all participants in our study avoided crowded places and close contact with other people. Most of them believed in the importance of using face masks when they go out during the pandemic process. Also, the Centers for Disease Control and Prevention (CDC) suggested wearing cloth face coverings to prevent transmission of the disease (16). Asymptomatic people who carry the virus but do not show symptoms of the disease can also cause the disease to spread (17). When we asked the question of whether people with COVID-19 can transmit the virus to others when they do not have a fever and cough, $66 \%$ of the patients answered correctly. With this question we asked, we have informed our patients who could not answer correctly. Our study indicated a higher level of appropriate hygienic practices among participants. Nearly all of the participants reported washing their hands with soap regularly for at least twenty seconds to prevent for transmission. Quarantine provides prevent spread of disease. CDC recommended quarantine period of 14 days for someone in contact with the coronavirus. When we asked our participants about their knowledge regarding quarantine day, most participants (95.5\%) answered correctly. Although there is no study in the literature directly questioning the covid quarantine period, when we scanned the literature, 96 $\%$ of the participants in a study conducted in India stated that quarantine is important (13).

\section{COVID-19 and Cancer}

Cancer patients constitute a particular group in the entire population. Since the treatment and follow-up of these patients had to continue, their constant coming to the hospital increased the risk of contamination. There wasn't any other study that demonstrated the knowledge and attitudes of cancer patients about COVID-19 when we had planned this study. That is the reason why we thought this kind of study should be done. Later, a similar study which we observed with pleasure was conducted by Erdem D. and Karaman I.(12). In the study by Dilek E. and Karaman I., a total of 13 questions were prepared and they were compared with demographic characteristics such as age, gender, education level. However, our study, as we mentioned before, consists of 25 questions in total and 3 parts. And we calculated the knowledge score to make the survey more objective. In addition to looking at the knowledge and attitudes of the patients, cancer patients' views between COVID-19 and cancer disease were also questioned. Both studies had general knowledge questions about COVID-19 and generally, knowledge levels were found to be positively high in both studies. In the study conducted by Erdem D. and Karaman I., 98\% of the patients participating in the survey did not postpone their cancer treatment (12). In our own study, we asked patients if they thought about delaying cancer treatments and half of the patients did not think delaying their treatments.

The majority of the participants thought that their cancer is a situation that increases the seriousness of this infection if they have COVID-19 disease. This result has been demonstrated from multiple studies published about the disease in China. As a result of this study conducted in China, the mortality rate of patients with COVID-19 and cancer was about 3-fold higher than those with COVID-19 without cancer. However patients with cancer and COVID-19 also tend to have much higher severity of their illness $(8,18)$. While half of the participants thought that COVID-19 outbreak did not prevent the treatment of cancer patients from 
continuing, $27 \%$ of patients thought that they could block their treatment. Within the recommendation of current oncology guidelines (e.g., ESMO, NCCN), in our oncology clinic, we extended the follow-up care for control patients who did not receive active treatment. We have arranged the chemotherapy protocols of our patients who receive active treatment in such a way that they should at least come to the hospital. When we asked the question of whether the patients thought about delaying their treatments, $70 \%$ of the patients did not think delaying their treatments. When asked whether your oncology doctor informed you about COVID-19 disease, $48.6 \%$ of patients said they informed us, $42 \%$ said they did not inform us. This may be because patients are unable to attend their doctor's appointment because of the pandemic. In addition, it also shows that as oncology doctors, we need to focus more on information about COVID-19 in our busy work. Among our cancer patients surveyed, we found that all of participants wore masks when they left the house during the outbreak. In another question we asked about protection from COVID-19, about $95 \%$ of patients stated they were paying attention to social distance with other people. CDC recommended that to practice social distancing, stay at least 6 feet (about two meters) about from other people who are not from your household in both indoor and outdoor spaces. While majority of the patients $(82.7 \%)$ thought that they took the necessary precautions to protect against the any disease, $15 \%$ stated that they applied partially. When we asked the question of whether the patients took additional protective or supportive products because of your cancer during the outbreak, sixty-one percent of participants expressed that they did not receive supportive products. There is no miraculous supplement that protects against COVID-19, which has been cited by the World Health Organization so far.

We asked about performing follow-up care in cancer patients during the COVID-19 pandemic period, half of the patients replied, if they do not have any symptoms, they would prefer to have it done when their doctor recommends. However about forty percent of patients also stated that they wanted to have control tests done on time. While half of patients were concerned about the continuation of cancer treatment during the outbreak, half of them expressed no concern. The majority of those who were concerned expressed that they were more afraid of the COVID-19 transmission than disruption of their cancer treatments. This means that the COVID-19 outbreak has significantly worried cancer patients. But patients also don't want their cancer treatments to run out. Therefore, it is very important that doctors keep in close contact with their patients.

\section{Limitations}

The study is limited to the only in one month period. This population doesn't represents the whole our patient population. Educated population of the region, especially don't come to routine follow-up if they have no symphtoms, so it should not be generalized to the whole population. The other limitation of this study is about quastionnaire, because we wan't to learn our patients thinkings about COVID-19 with cancer, so some of the questions we write for patients to choose have two choice and some of them have three choice. This caused some difficulties for calculating the knowledge score. We had to exclude some interpretative questions from the knowledge score.

\section{Conclusion}

We conducted a study evaluating the awareness, knowledge and perceptions of cancer patients during the COVID-19 outbreak. It is important to evaluate these parameters in risky cancer patient population for informing patients about COVID-19 for preventing of this mortal virus infection.

\section{Financial support}

None.

\section{Conflicts of Interest}

None declared.

\section{Acknowledgement}

None. 


\section{Hosted file}

table 1.pdf available at https://authorea.com/users/386186/articles/501563-evaluation-ofknowledge-attitudes-and-behaviours-about-covid-19-among-cancer-patients

\section{Hosted file}

Table 2.pdf available at https://authorea.com/users/386186/articles/501563-evaluation-ofknowledge-attitudes-and-behaviours-about-covid-19-among-cancer-patients

\section{Hosted file}

Table 3.pdf available at https://authorea.com/users/386186/articles/501563-evaluation-ofknowledge-attitudes-and-behaviours-about-covid-19-among-cancer-patients

\section{Hosted file}

Table 4.1.pdf available at https://authorea.com/users/386186/articles/501563-evaluation-ofknowledge-attitudes-and-behaviours-about-covid-19-among-cancer-patients

\section{Hosted file}

Table 4.2.pdf available at https://authorea.com/users/386186/articles/501563-evaluation-ofknowledge-attitudes-and-behaviours-about-covid-19-among-cancer-patients

\section{Hosted file}

Table 5.pdf available at https://authorea.com/users/386186/articles/501563-evaluation-ofknowledge-attitudes-and-behaviours-about-covid-19-among-cancer-patients 